\title{
Zebrafish Embryo and Acute Fish Toxicity Test Show Similar Sensitivity for Narcotic Compounds
}

\author{
Anita Birke 1 and Stefan Scholz 2 \\ ${ }^{1}$ BioChem agrar, Machern, Germany, work partially conducted as a visiting scientist at the UFZ; ${ }^{2}$ Helmholtz Centre for Environmental Research - UFZ, \\ Department of Bioanalytical Ecotoxicology, Leipzig, Germany
}

\begin{abstract}
The zebrafish embryo test has been discussed as an alternative test system to provide data on acute fish toxicity required by diverse regulations. A meta-analysis of zebrafish embryo acute toxicity (ZFET) data has revealed conflicting evidence that narcotic compounds (i.e., compounds with baseline toxicity) may exhibit weaker sensitivity in the ZFET compared to the acute (adult) fish toxicity test (AFT). Therefore, six compounds with presumably narcotic or unknown mode of action, and for which a previous meta-analysis had indicated weaker sensitivity, were experimentally analyzed for their fish embryo acute toxicity and exposure concentrations were monitored. The data indicated that ZFET and AFT for the selected compounds had similar sensitivity and differences were in the range of species differences of the AFT.
\end{abstract}

\section{Introduction}

The estimation of acute fish toxicity is required worldwide by diverse regulations for the hazard and risk assessment of chemicals, pesticides, biocides, (veterinary) drugs, and feed additives (reviewed in Scholz et al., 2013). Typically, this information is provided by experimental data based on the acute exposure of fish for $96 \mathrm{~h}$ to the test chemicals according to an internationally agreed testing guideline (OECD, 1992). The fish embryo test has been suggested as one potential alternative approach to reduce the number of tests using juvenile or adult fish (reviewed in Scholz et al., 2013), since the fish embryo is considered a non-protected life stage by some animal welfare regulations (Halder et al., 2010; Embry et al., 2010). In the case of zebrafish, which is one of the most commonly used model organisms for fish embryo tests, stages up to $5 \mathrm{dpf}$ (days post fertilization) are commonly used in fish embryo toxicity tests (Strähle et al., 2012). Various meta- and experimental analyses have revealed a high correlation of zebrafish acute embryo toxicity (ZFET) with acute fish toxicity (AFT) with on average similar sensitivity (Lammer et al., 2009a; Busquet et al., 2014; Belanger et al., 2013). The on average high correlation and sensitivity as well as the high reproducibility has led to establishment of the OECD testing guideline 236: "Fish Embryo Acute Toxicity (FET) Test" (OECD, 2013; Busquet et al., 2014).
However, concern has been raised about the applicability domain of the ZFET, given that, for example, neurotoxic compounds appear to exhibit no or weak acute toxicity in fish embryos (Klüver et al., 2015; Sobanska et al., 2018; Glaberman et al., 2016). The weak toxicity of neurotoxic compounds in fish embryos was explained by the lack of respiratory failure syndrome in fish embryos due to uptake of oxygen by diffusion and it being independent of the function of the cardiovascular system. Given that neurotoxic compounds are likely to impact on behavior, it was suggested to assess embryonic movement to identify neurotoxic compounds in order to predict their acute toxicity in later life stages (Klüver et al., 2015). Conflicting evidence was provided for the applicability of the ZFET for presumably narcotic (baseline toxic) compounds. A QSAR established for ZFET baseline toxicity was very similar to the available AFT QSARs (Klüver et al., 2016). However, the meta-analysis of Sobanska et al. (2018) identified 9 out of 47 narcotic compounds that exhibited an LC50 that was more than 10-fold higher than the AFT LC50 in one of four species used for comparison (zebrafish, rainbow trout, fathead minnow, bluegill). For this meta-analysis, a database of 153 study entries representing 123 compounds with corresponding AFTs was established. Given the lack of studies that had been conducted strictly according to the OECD TG 236, the database also considered studies performed similarly to the TG 236. To avoid bias by studies deviating from TG 236, certain
Received August 10, 2018; Accepted October 29, 2018; Epub October 29, 2018; (C) The Authors, 2018.

ALTEX 36(1), 131-135. doi:10.14573/altex.1808101

Correspondence: Stefan Scholz, PhD, Helmholtz Centre for Environmental Research - UFZ, Dpt. of Bioanalytical Ecotoxicology, Permoserstr. 15,

04318 Leipzig, Germany

(stefan.scholz@ufz.de)
This is an Open Access article distributed under the terms of the Creative Commons Attribution 4.0 International license (http://creativecommons.org/licenses/by/4.0/) which permits unrestricted use, distribution and reproduction in any medium, provided the original work is appropriately cited. 
quality criteria were considered, i.e., only studies were accepted that had the LC50 inside the water solubility range, exposure duration from 96 to $120 \mathrm{~h}$, a predicted, buffered or measured $\mathrm{pH}$ range in exposure solutions between 5-9, a concentration range including baseline toxicity for non-toxic compounds, and a log $K_{\text {ow }}<4$ if exposure concentrations were not measured (Sobanska et al. 2018). We hypothesized that part of the discrepancy may be due to experimental limitations, since ZFET data included in the meta-analysis were often derived from studies of small scale high-throughput testing that lacked verification of exposure concentrations. Therefore, we selected six compounds for which a weak toxicity in the ZFET had been previously reported for experimental verification by conducting a ZFET according to OECD TG 236 (OECD, 2013) including chemical analysis of exposure solutions. The compounds were selected from the previous meta-analysis (Sobanska et al., 2018) according to two criteria: availability of analytical methods and differences in hydrophobicity that span a wide range. Five of the chosen compounds exhibit a narcotic mode of action (MoA, 4-chloroaniline, aniline, acetochlor, folpet, pyraclostrobin); for one compound (3-iodo-2-propynyl-N-butylcarbamate) the MoA is unknown.

\section{Animals, materials and methods}

The zebrafish embryo acute toxicity test was conducted according to the TG 236 with minor modifications. Details on all materials and methods including methods used for the analysis of exposure media (Tab. S1), and physico-chemical properties of exposure chemicals and measured concentration of exposure solutions (Tab. S2) can be found in supplementary file $1^{1}$. Exposure concentrations were measured for all test chemicals using HPLC-MSMS. Origin of acute fish toxicity data is given in supplementary file $2^{2}$.

\section{Results and discussion}

\subsection{Analysis of exposure concentrations}

It has been recognized that exposure concentrations can drop during incubation in plastic microtiter plates owing to adsorption of hydrophobic compounds or compound volatilization. This can affect the estimation of effect concentrations when test organisms are incubated in plastic microtiter plates as is the case in the ZFET (Schreiber et al., 2008; Riedl and Altenburger, 2007). In line with this, the meta-analysis of FET data by Sobanska et al. (2018) indicated that for substances with a $\log K_{\text {ow }}>4$ and a $\log K_{\text {aw }}>-4$ often no mortality was observed (Sobanska et al., 2018). Loss of test compound would result in an overestimation of effect concentration, particularly if it was determined based on nominal concentration. To avoid this, pre-saturation of test plates $24 \mathrm{~h}$ prior to the start of the exposure and/or determination of the effect concentration based on the measured concentration was recommended by the OECD TG 236 (OECD, 2013). Measured concentrations are not available for most of the fish embryo and also many acute fish tests conducted to date, which may account for some of the observed discrepancies between AFT and ZFET data (Sobanska et al., 2018).

However, in the present study we did not observe any drop of exposure concentrations that could be associated with adsorption of the test compound to microtiter plates. Chemical analysis indicated stable exposure concentrations (i.e., deviation of less than $20 \%$ from the concentration at the start of the exposure) for all substances except folpet. Folpet is known to be rapidly hydrolyzed with a reported half-life of $0.7 \mathrm{~h}\left(\mathrm{pH} 7,25^{\circ} \mathrm{C}\right.$; EFSA, 2009). Therefore, the stability of folpet was monitored initially in exposure medium for $7 \mathrm{~h}$. As expected, the concentration dropped below the detection limit of $0.012 \mu \mathrm{M}$ within $5 \mathrm{~h}$ and a half-life of 29 minutes was calculated (Fig. S1 ${ }^{1}$ ). Accordingly, optimal testing of folpet would require a flow-through system, such as proposed by Lammer et al. (2009b). However, in order to compensate at least partially for the hydrolytic loss, a semi-static exposure with 12-h renewal intervals was used. For pyraclostrobin, chemical analysis revealed exposure concentrations below $80 \%$ of the nominal concentrations already at the start of the exposure (Tab. S3 ${ }^{1}$ ). However, the concentration remained stable over the exposure duration, excluding adsorption to the microwell plates. Instead, incomplete dissolution may account for the lower measured concentration. Therefore, as recommended by the OECD, the geometric mean of measured concentrations detected at the beginning and end of the exposure interval was used to derive effect concentrations (OECD, 2013). Due to the rapid drop below the detection limit within the 12-h renewal interval no geometric mean could be calculated for folpet. Hence, the nominal concentrations were used to derive the LC50 concentrations and it is likely that effect concentrations are thus overestimated for this compound.

\subsection{Determination of effect concentrations}

For all compounds a concentration-dependent increase in mortality approaching a $100 \%$ mortality level was observed and could be used to model concentration-response curves and calculate LC50 values (Tab. 1, Fig. S2 ${ }^{1}$ ). The effect concentrations decreased with increasing exposure time, particularly within the first $48 \mathrm{~h}$ of exposure (Tab. 1, Fig. S2 ${ }^{1}$ ). Given that for the selected compounds death was generally caused by coagulation, this indicates that the internal equilibrium concentration may have only been approached at the end of the exposure. Observations that for many compounds no equilibrium internal concentrations had been established after $24 \mathrm{~h}$ of exposure have been reported previously (e.g., El-Amrani et al., 2012; Brox et al., 2014, 2016). The observed toxic ratio (TR, ratio of predicted baseline versus observed LC50) confirmed the classification of aniline, acetochlor, and pyraclostrobin as narcotic compounds (i.e., compounds with toxicity in the range of the calculated baseline tox-

\footnotetext{
1 doi:10.14573/altex.1808101s1

2 doi:10.14573/altex.1808101s2
} 
Tab. 1: LC50 with confidence intervals, modelling parameters and comparative baseline and acute toxicity data Previously published LC50 for zebrafish embryos (ZFET) and acute fish toxicity (AFT) were obtained from the meta-analysis of Sobanska et al. (2018); individual references and test conditions are given in Tab. S41) and refer to exposure durations of 96 or $120 \mathrm{~h}$. DR, Danio rerio (zebrafish); LM, Lepomis macrochirus (bluegill); OM, Oncorhynchus mykiss (rainbow trout); PP, Pimephales promelas (fathead minnow).

\begin{tabular}{|c|c|c|c|c|c|c|c|c|}
\hline Substance (MoA) & $\begin{array}{l}\mathrm{LC}_{50} 24 \text { hpf } \\
(\mu \mathrm{mol} / \mathrm{l})\end{array}$ & $\begin{array}{l}L^{L} C_{50} 48 \text { hpf } \\
(\mu \mathrm{mol} / \mathrm{l})\end{array}$ & $\begin{array}{l}L^{L} C_{50} 72 \text { hpf } \\
(\mu \mathrm{mol} / \mathrm{l})\end{array}$ & $\begin{array}{l}L_{50} 96 \text { hpf } \\
(\mu \mathrm{mol} / \mathrm{I})\end{array}$ & $\begin{array}{l}\text { FET-LC }_{50^{-}} \\
\text {values } \\
\text { ( } \mu \mathrm{mol} / \mathrm{I}) \\
\text { from meta- } \\
\text { analysis }\end{array}$ & $\begin{array}{l}\text { ZFET } \\
\text { baseline } \\
\text { LC50 } \\
(\mu \mathrm{mol} / \mathrm{l})\end{array}$ & TR (ZFET) & $\begin{array}{l}\text { ZFET/AFT } \\
\text { ratio }\end{array}$ \\
\hline $\begin{array}{l}\text { 4-Chloroaniline } \\
\text { (Narcosis) } \\
\text { Slope }\end{array}$ & $\begin{array}{l}291 \\
(267-314) \\
8.43\end{array}$ & $\begin{array}{l}194 \\
(182-206) \\
6.14\end{array}$ & $\begin{array}{l}164 \\
(158-169) \\
6.53\end{array}$ & $\begin{array}{l}151 \\
(140-162) \\
5.21\end{array}$ & $\begin{array}{l}345 \\
\text { (Burkhardt- } \\
\text { Holm et al., } \\
1999)\end{array}$ & 2076 & 14 & \begin{tabular}{|l|}
$0.55(\mathrm{DR})$ \\
$8.0(\mathrm{LM})$ \\
$1.4(\mathrm{OM})$ \\
$0.84(\mathrm{PP})$
\end{tabular} \\
\hline $\begin{array}{l}\begin{array}{l}\text { Acetochlor } \\
\text { (Narcosis) }\end{array} \\
\end{array}$ & $\begin{array}{l}126 \\
(107-145) \\
7.34\end{array}$ & $\begin{array}{l}72.8 \\
(40.3-105) \\
67\end{array}$ & \begin{tabular}{|l|}
53.2 \\
$(47.4-58.9)$ \\
13.2
\end{tabular} & $\begin{array}{l}34.6 \\
(32.2-37.1) \\
6.19\end{array}$ & $\begin{array}{l}57.7 \\
\text { (Truong et al., } \\
2014 \text { ) } \\
26.6 \\
\text { (Padilla et al., } \\
2012 \text { ) }\end{array}$ & 48.3 & 1.4 & \begin{tabular}{|l|}
6.5 (LM) \\
16 (OM)
\end{tabular} \\
\hline $\begin{array}{l}\text { Aniline } \\
\text { (Narcosis) }\end{array}$ & $\begin{array}{l}1910 \\
(615-3210) \\
24\end{array}$ & \begin{tabular}{l|}
1790 \\
$(1590-1990)$ \\
4.73
\end{tabular} & \begin{tabular}{|l|}
1720 \\
$(1450-1990)$ \\
3.24
\end{tabular} & $\begin{array}{l}1660 \\
(1430-1880) \\
3.32\end{array}$ & $\begin{array}{l}9300 \\
\text { (Groth et al., } \\
1993 \text { ) }\end{array}$ & 8929 & 5.4 & $\begin{array}{l}2.6(\mathrm{DR}) \\
3.2(\mathrm{LM}) \\
4.7(\mathrm{OM}) \\
1.8(\mathrm{PP})\end{array}$ \\
\hline $\begin{array}{l}\text { 3-lodo-2-propynyl- } \\
\text { N-butylcarbamate } \\
\text { (Unknown) Slope }\end{array}$ & $\begin{array}{l}1.44 \\
(1.38-1.5) \\
8.56\end{array}$ & $\begin{array}{l}1.35 \\
(1.25-1.45) \\
9.62\end{array}$ & $\begin{array}{l}1.34 \\
(1.25-1.42) \\
11.5\end{array}$ & $\begin{array}{l}1.34 \\
(1.25-1.42) \\
11.5\end{array}$ & $\begin{array}{l}2.55 \\
\text { (Padilla et al., } \\
2012 \text { ) } \\
20.2 \\
\text { (Truong et al., } \\
2014 \text { ) }\end{array}$ & 393 & 293 & $\begin{array}{l}1.7 \text { (LM) } \\
4.8(\mathrm{OM}) \\
1.9(\mathrm{PP})\end{array}$ \\
\hline $\begin{array}{l}\text { Pyraclostrobin* } \\
\text { (Narcosis) } \\
\text { Slope }\end{array}$ & $\begin{array}{l}0.264 \\
(0.239- \\
0.288) \\
8.94\end{array}$ & $\begin{array}{l}0.184 \\
(0.152- \\
0.216) \\
70.3\end{array}$ & \begin{tabular}{|l|}
0.178 \\
$(0.176-$ \\
$0.181)$ \\
36.2
\end{tabular} & $\begin{array}{l}0.138 \\
(0.122- \\
0.154) \\
3.69\end{array}$ & $\begin{array}{l}0.187 \\
\text { (Padilla et al., } \\
2012 \text { ) } \\
0.498 \\
\text { (Truong et al., } \\
2014 \text { ) }\end{array}$ & 0.43 & 3.1 & $\begin{array}{l}4.7 \text { (LM) } \\
8.6(\mathrm{OM})\end{array}$ \\
\hline $\begin{array}{l}\text { Folpet } \\
\text { (Narcosis) }\end{array}$ & $\begin{array}{l}3.78 \\
(3.39-4.17) \\
8.97\end{array}$ & $\begin{array}{l}2.7 \\
(2.32-3.08) \\
4.92\end{array}$ & \begin{tabular}{|l|}
2.47 \\
$(2.18-2.77)$ \\
9.35
\end{tabular} & $\begin{array}{l}2.09 \\
(1.31-2.86) \\
20.3\end{array}$ & $\begin{array}{l}8.88 \\
\text { (Padilla et al., } \\
\text { 2012) }\end{array}$ & 162 & 77 & \begin{tabular}{|l}
$8.5(\mathrm{LM})$ \\
$9.9(\mathrm{OM})$ \\
$3.4(\mathrm{PP})$
\end{tabular} \\
\hline
\end{tabular}

*based on measured exposure concentrations

icity). A slightly enhanced TR was observed for 4-chloroaniline, which may indicate that this compound does not act through baseline toxicity but also exhibits a specific mode of action. Furthermore, the TR of folpet (77) supports a potential specific or reactive mode of action for this compound.

When the 96-h LC50s were compared to previous zebrafish embryo studies (Tab. 1), lower effect concentrations were obtained for nearly all compounds. The maximum differences ranged from 1.4-fold (3-iodo-2-propynyl-N-butylcarbamate) to 5.6-fold (aniline). Only for acetochlor slightly higher effect concentrations were obtained in our study compared to the study of Padilla et al. (2012). Due to this increased sensitivity, all derived effect concentrations were closer to the range observed for AFT in rainbow trout, fathead minnow, bluegill, or zebrafish (Tab. 1). The relative effect concentration $\left(\mathrm{LC} 50_{\mathrm{ZFET}} / \mathrm{LC} 50_{\mathrm{AFT}}\right)$ of the ZFET ranged from 0.55 (4-chloroaniline) to 16 (acetochlor) and was also dependent on the species that was used for the com- parative AFT value. For instance, relative effect concentrations of ZFET and exposure to acetochlor were lower compared to the LC50 $0_{\mathrm{AFT}}$ of bluegill (6.4). The on average slightly higher effect concentrations for ZFET may relate to species sensitivity rather than stage-specific sensitivities. This is indicated by the two compounds (4-chloroaniline, aniline), for which a zebrafish LC50 $0_{\mathrm{AFT}}$ was available and was closer to the LC50 ${ }_{\mathrm{ZFET}}$ than the LC50s of other species. A factor of 10 - based on mean species differences of the AFT - has been previously selected as a threshold to indicate whether ZFET data is exhibiting a weaker sensitivity (Sobanska et al., 2018). If this factor is applied to the data obtained in the present study, none of the compounds is classified as a compound with weaker sensitivity in the ZFET. Only for aniline, a weaker sensitivity may be assigned if data are compared to the LC50 $\mathrm{AFT}$ of bluegill.

It is difficult to conclude why a higher sensitivity was obtained for nearly all compounds in our study since for previous studies 
no data on measured concentrations were available. In case of pyraclostrobin, the deviation of measured from nominal concentrations and the use of measured concentrations for calculation of the LC50 may have contributed to the difference in sensitivity $\left(\mathrm{Tab} . \mathrm{S}^{1}\right.$ ). For other compounds, the low exposure volumes (100 compared to $250 \mu \mathrm{l}$ ) in previous studies (Padilla et al., 2012; Truong et al., 2014) could have led to a drop of exposure concentrations due to accumulation in the fish embryo, particularly for compounds with higher hydrophobicity. Plastic polystyrene microtiter plates were used in the previous as well as in our study and the measured concentration did not indicate that adsorption to microplates accounted for the sensitivity differences. Finally, a rapid loss of compound due to, e.g. hydrolysis, may be partially compensated by the frequent renewal of exposure solutions. This was indicated by similar effect concentrations obtained for folpet compared to AFT data. However, the relevance of such compounds for acute exposure scenarios should be questioned or appropriate exposure protocols to maintain stable exposure concentrations should be developed.

\section{Conclusions}

The previously observed weaker sensitivity of ZFET for some presumably narcotic compounds and one compound with an unknown mode of action was not found to the same extent in this study. Effect concentrations within a factor of 10 of the LC $50_{\text {AFT }}$ were obtained for all studied compounds. Given the lack of chemical analysis data in the previous study, it was difficult to conclude whether the higher sensitivity observed in this study was due to more stable exposure concentrations, and/or the use of measured concentration (in case of deviation from nominal) for calculation of the LC50. The study confirms that, as previously suggested by quantitative structure activity relationships, a similar sensitivity can be expected for narcotic compounds for the baseline toxicity in fish embryos and juvenile/adult fish.

\section{References}

Belanger, S. E., Rawlings, J. M. and Carr, G. J. (2013). Use of fish embryo toxicity tests for the prediction of acute fish toxicity to chemicals. Environ Toxicol Chem 32, 1768-1783. doi:10.1002/etc. 2244

Brox, S., Ritter, A. P., Küster, E. and Reemtsma, T. (2014). A quantitative HPLC-MS/MS method for studying internal concentrations and toxicokinetics of 34 polar analytes in zebrafish (Danio rerio) embryos. Anal Bioanal Chem 406, 4831-4840. doi:10.1007/s00216-014-7929-y

Brox, S., Seiwert, B., Küster, E. and Reemtsma, T. (2016). Toxicokinetics of polar chemicals in zebrafish embryo (Danio rerio): Influence of physico-chemical properties and of biological processes. Environ Sci Technol 50, 10264-10272. doi:10.1021/acs.est.6b04325

Burkhardt-Holm, P., Oulmi, Y., Schroeder, A. et al. (1999). Toxicity of 4-chloroaniline in early life stages of zebrafish
(Danio rerio): II. Cytopathology and regeneration of liver and gills after prolonged exposure to waterborne 4-chloroaniline. Arch Environ Contam Toxicol 37, 85-102. doi:10.1007/ s002449900493

Busquet, F., Strecker, R., Rawlings, J. M. et al. (2014). OECD validation study to assess intra- and inter-laboratory reproducibility of the zebrafish embryo toxicity test for acute aquatic toxicity testing. Regul Toxicol Pharmacol 69, 496-511. doi:10.1016/j.yrtph.2014.05.018

EFSA (2009). Conclusion on Pesticide Peer Review. Peer review of the pesticide risk assessment of the active substance folpet (Question No: EFSA-Q-2009-605). EFSA Journal 297. doi:10.2903/j.efsa.2009.297r

El-Amrani, S., Pena-Abaurrea, M., Sanz-Landaluze, J. et al. (2012). Bioconcentration of pesticides in zebrafish eleutheroembryos (Danio rerio). Sci Total Environ 425, 184-190. doi:10.1016/j.scitotenv.2012.02.065

Embry, M. R., Belanger, S. E., Braunbeck, T. A. et al. (2010). The fish embryo toxicity test as an animal alternative method in hazard and risk assessment and scientific research. Aquat Toxicol 97, 79-87. doi:10.1016/j.aquatox.2009.12.008

Glaberman, S., Padilla, S. and Barron, M. G. (2016). Evaluating the zebrafish embryo toxicity test for pesticide hazard screening. Environ Toxicol Chem 36, 1221-1226. doi:10.1002/ etc. 3641

Groth, G., Schreeb, K., Herdt, V. and Freundt, K. J. (1993). Toxicity studies in fertilized zebrafish eggs treated with N-methylamine, N,N-dimethylamine, 2-aminoethanol, isopropylamine, aniline, N-methylaniline, N,N-dimethylaniline, quinone, chloroacetaldehyde, or cyclohexanol. Bull Environ Contam Toxicol 50, 878-882. doi:10.1007/BF00209953

Halder, M., Léonard, M., Iguchi, T. et al. (2010). Regulatory aspects on the use of fish embryos in environmental toxicology. Integr Environ Assess Manag 6, 484-491. doi:10.1002/ ieam. 48

Klüver, N., König, M., Ortmann, J. et al. (2015). Fish embryo toxicity test: Identification of compounds with weak toxicity and analysis of behavioral effects to improve prediction of acute toxicity for neurotoxic compounds. Environ Sci Technol 49, 7002-7011. doi:10.1021/acs.est.5b01910

Klüver, N., Vogs, C., Altenburger, R. et al. (2016). Development of a general baseline toxicity QSAR model for the fish embryo acute toxicity test. Chemosphere 164, 164-173. doi:10.1016/j. chemosphere.2016.08.079

Lammer, E., Carr, G. J., Wendler, K. et al. (2009a). Is the fish embryo toxicity test (FET) with the zebrafish (Danio rerio) a potential alternative for the fish acute toxicity test? Comp Biochem Physiol C Toxicol Pharmacol 149, 196-209. doi:10.1016/j.cbpc.2008.11.006

Lammer, E., Kamp, H. G., Hisgen, V. et al. (2009b). Development of a flow-through system for the fish embryo toxicity test (FET) with the zebrafish (Danio rerio). Toxicol In Vitro 23, 1436-1442 doi:10.1016/j.tiv.2009.05.014

OECD (1992). Test No. 203: Fish, Acute Toxicity Test. OECD Guidelines for the Testing of Chemicals, Section 2. OECD Publishing, Paris. doi:10.1787/9789264069961-en 
OECD (2013). Test No. 236: Fish Embryo Acute Toxicity (FET) Test. OECD Guidelines for the Testing of Chemicals, Section 2. OECD Publishing, Paris. doi:10.1787/9789264203709-en

Padilla, S., Corum, D., Padnos, B. et al. (2012). Zebrafish developmental screening of the ToxCast ${ }^{\mathrm{TM}}$ Phase I chemical library. Reprod Toxicol 33, 174-187. doi:10.1016/j.reprotox. 2011.10.018

Riedl, J. and Altenburger, R. (2007). Physicochemical substance properties as indicators for unreliable exposure in microplate-based bioassays. Chemosphere 67, 2210-2220. doi:10.1016/j.chemosphere.2006.12.022

Scholz, S., Sela, E., Blaha, L. et al. (2013). A European perspective on alternatives to animal testing for environmental hazard identification and risk assessment. Regul Toxicol Pharmacol 67, 506-530. doi:10.1016/j.yrtph.2013.10.003

Schreiber, R., Altenburger, R., Paschke, A. and Küster, E. (2008). How to deal with lipophilic and volatile organic substances in microtiter plate assays. Environ Toxicol Chem 27, 1676-1682. doi:10.1897/07-504.1

Sobanska, M., Scholz, S., Nyman, A.-M. et al. (2018). Applicability of the fish embryo acute toxicity (FET) test (OECD 236) in the regulatory context of REACH. Environ Toxicol Chem 37, 657-670. doi:10.1002/etc.4055

Strähle, U., Scholz, S., Geisler, R. et al. (2012). Zebrafish embryos as an alternative to animal experiments - A commentary on the definition of the onset of protected life stages in animal welfare regulations. Reprod Toxicol 33, 128-132. doi:10.1016/j.reprotox.2011.06.121

Truong, L., Reif, D. M., St Mary, L. et al. (2014). Multidimensional in vivo hazard assessment using zebrafish. Toxicol Sci 137, 212-233. doi:10.1093/toxsci/kft235

\section{Conflict of interest}

The authors declare that they have no conflict of interest.

\section{Acknowledgements}

We thank Dr Winkler BioChem agrar for his support and for providing the possibility to conduct the analysis in the laboratories of BioChem agrar. Nicole Schweiger, UFZ is acknowledged for providing fish embryos. 